\title{
Development of high gradient laser wakefield accelerators towards nuclear detection applications at LBNL
}

\author{
Cameron G.R. Geddes ${ }^{1}$, David L. Bruhwiler ${ }^{2}$, John R. Cary ${ }^{2,3}$, Eric H. Esarey ${ }^{1,4}$, \\ Anthony J. Gonsalves ${ }^{1}$, Chen Lin $^{1}$, Estelle Cormier-Michel ${ }^{1}$, Nicholas H. Matlis ${ }^{1}$, \\ Kei Nakamura $^{1,4}$, Mike Bakeman ${ }^{1,4}$, Dmitriy Panasenko ${ }^{1}$, Guillaume R. Plateau ${ }^{1}$, \\ Carl B. Schroeder ${ }^{1}$, Csaba Toth ${ }^{1}$, Wim P. Leemans ${ }^{1,4}$ \\ ${ }^{1}$ LOASIS Program, LBNL, 1 Cyclotron Rd MS 71-259, Berkeley CA 94720, United States \\ ${ }^{2}$ Tech-X Corp., 5621 Arapahoe Ave Ste. A, Boulder CO 80303, United States \\ ${ }^{3}$ U. Colorado, Boulder CO, United States \\ ${ }^{4}$ U. Nevada Reno, Reno NV, United States
}

\begin{abstract}
Compact high-energy linacs are important to applications including monochromatic gamma sources for nuclear material security applications. Recent laser wakefield accelerator experiments at LBNL demonstrated narrow energy spread beams, now with energies of up to $1 \mathrm{GeV}$ in $3 \mathrm{~cm}$ using a plasma channel at low density. This demonstrates the production of $\mathrm{GeV}$ beams from devices much smaller than conventional linacs, and confirms the anticipated scaling of laser driven accelerators to $\mathrm{GeV}$ energies. Stable performance at $0.5 \mathrm{GeV}$ was demonstrated. Experiments and simulations are in progress to control injection of particles into the wake and hence to improve beam quality and stability. Using plasma density gradients to control injection, stable beams at $1 \mathrm{MeV}$ over days of operation, and with an order of magnitude lower absolute momentum spread than previously observed, have been demonstrated. New experiments are post-accelerating the beams from controlled injection experiments to increase beam quality and stability. Thomson scattering from such beams is being developed to provide collimated multi-MeV monoenergetic gamma sources for security applications from compact devices. Such sources can reduce dose to target and increase accuracy for applications including photofission and nuclear resonance fluorescence.
\end{abstract}

Keywords: laser wakefield acceleration, X-rays, Thomson scattering.

PACS: $52.38 . \mathrm{Kd}, 07.77 . \mathrm{Ka} ; 07.85 . \mathrm{Fv}$

\section{INTRODUCTION}

Monoenergetic sources allow special nuclear materials (SNM) signatures to be obtained with greatly reduced radiation doses to the target compared to broadband Bremsstrahlung sources. In addition to reduced dose, the narrow energy spread reduces background, which could improve detection in nuclear resonance fluorescence (NRF) based cargo screening, where a very narrow linewidth NRF signal must be detected. Thomson scattering of relativistic electrons and laser photons produces a suitable beam of nearly monoenergetic gamma-rays. At $180^{\circ}$ the Thomson up-scattered photons are produced at an energy related to the electron energy and laser energy by: $\mathrm{h} v_{\text {scatter }}=4 \gamma^{2} \mathrm{~h} v_{\text {laser }}$ where $\gamma \sim 2 \mathrm{E}_{\text {electron }}[\mathrm{MeV}] . \quad$ The technique has been developed on conventional linacs [1], and scattering of $0.5-1 \mu \mathrm{m}$ laser light (produced by high power solid state lasers) from electron beams at energies of $200-800 \mathrm{MeV}$ produces photons of 1.7 - $15 \mathrm{MeV}$ suitable for NRF or photofission interrogation. Divergence is $\sim \operatorname{mrad}$ (a convolution of electron divergence and Thomson broadening), allowing projection of a $\mathrm{cm}$-size spot at $100 \mathrm{~m}$ standoff for remote detection. Novel compact electron accelerators are needed because conventional accelerator lengths are order $10 \mathrm{~m}$ for energies needed to produce $1.7 \mathrm{MeV}$ photons for $\mathrm{NRF}$, and order $50 \mathrm{~m}$ long for energies needed for photofission. While conventional linac development is expected to reduce size somewhat, new technologies are needed to produce compact systems.

Laser-driven, plasma wakefield accelerators (LWFAs) [2] in use at LBNL provide high-quality electron beams with percent energy spread at the $0.2-$ $1 \mathrm{GeV}$ energies required for SNM detection with Thomson sources in $0.2-3 \mathrm{~cm}$ distances $[3,4]$. In such accelerators (Fig.1), an intense laser pulse drives a 
space charge wave in a plasma producing acceleration gradients on the order of $100 \mathrm{GV} / \mathrm{m}$, thousands of times those in conventional linacs. Electrons are trapped from the plasma and accelerated by the wave. Modern laser drivers of the required power for both drive laser and Thomson scattering are approximately $30 \mathrm{~m}^{2}$ combined and have shrunk several-fold in cost and size in the last four years. Continued laser and accelerator development hence may enable compact Thomson based SNM detection systems. Such accelerators are also important to other radiation sources, including free electron lasers [5] and $\mathrm{THz}$ [6] sources. The electron bunch, and hence the radiation pulse, are fs in duration due to the $\mu \mathrm{m}$ period of the plasma wave enabling diagnosis of ultrafast phenomena. This paper briefly discusses the beams achieved and use of controlled injection [7] and accelerator design techniques which are further improving energy spread and increasing stability. Applications to SNM radiation sources are outlined.

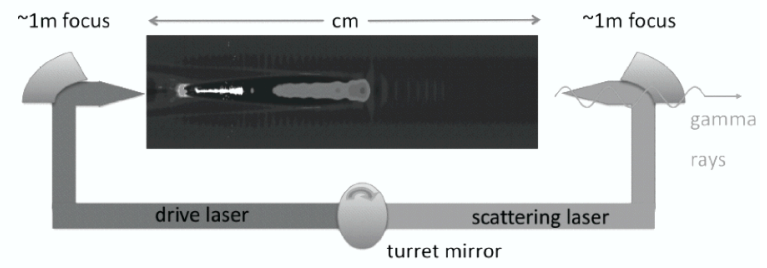

FIGURE 1. Schematic diagram of a LWFA and associated Thomson source, showing the laser driver (red/light grey) which drives a plasma wave wake (blue/dark grey) and accelerates an electron bunch (white), from a VORPAL particle simulation. The scattering laser is focused to intersect the electron bunch just after the accelerator (green/at right) producing gamma rays. The source is $\sim 2 \mathrm{~m}$ in scale facilitating rotation.

\section{LASER WAKEFIELD ACCELERATOR DEVELOPMENT}

Wakefield accelerator experiments at LBNL use the $10 \mathrm{~Hz}, 0.8 \mu \mathrm{m}$ wavelength LOASIS Ti:Sapphire laser [8], which has multiple arms delivering peak powers of 60 (12) TW for the main cryo (liquid) cooled arms. Low-stress crystal mounting and detailed optical optimization have resulted in near-diffractionlimited focal spots without deformable optics, including a $25 \mu \mathrm{m}$ spot with $\mathrm{M}^{2}=1.3$, and with $2.5 \mu \mathrm{rad}$ pointing stability, $3 \% \mathrm{rms}$ energy stability, and energies up to 2.3 Joules. The laser delivers pulses of the 30-50 fs durations required to resonantly drive the plasma wake, with $2.5 \% \mathrm{rms}$ variation in pulse length. Various spot sizes are obtained, producing intensities of $10^{18}-10^{19} \mathrm{~W} / \mathrm{cm}^{2}$.

Shaped plasma targets are used to guide the laser pulse in analogy to a plasma optical fiber. This extends the acceleration length, and resulted in the first production and measurement of high quality electron bunches from laser-plasma accelerators. Using a 10 TW laser, a narrow energy spread electron bunch was observed at $86 \pm 2 \mathrm{MeV}$ with $2 \times 10^{9}$ electrons within the narrow peak and with 3 mrad divergence [3] (similar results simultaneously reported in [9], and subsequently by others).

Channel-guided LWFA experiments at LBNL recently produced high quality electron bunches at 1 $\mathrm{GeV}$ [4]. Energy gain was extended to $\mathrm{GeV}$ using 40 TW laser power, longer plasma channels (e.g., 3.3 $\mathrm{cm}$ ), and lower plasma densities which extended the dephasing length by increasing the laser group velocity and hence the wake velocity (e.g., $10^{18} \mathrm{~cm}^{-3}$ ). A gasfilled capillary discharge waveguide [10] was used to produce the cm-scale, lower density plasma channels. Stable operation with few percent energy fluctuation and mrad pointing accuracy and divergence were obtained at $0.5 \mathrm{GeV}$ (Fig. 2) by tuning the timing of the discharge and laser, and the plasma density. New measurements with improved timing control now show percent stability over 10's of sequential shots [11]. Channel guiding can increase beam charge or energy for a given laser power compared to relying only on self-guiding of the laser pulse through relativistic effects.

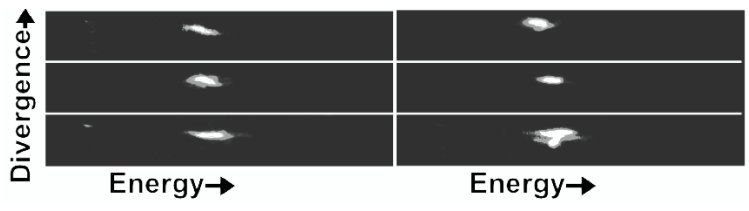

FIGURE 2. Six magnetic spectrometer shots from a stable regime at Energy $=456 \pm 45 \mathrm{MeV}, \mathrm{dE} / \mathrm{E}=6 \pm 3 \%$, and with Charge $=32 \pm 14 \mathrm{pC}\left(2 \times 10^{8}\right.$ electrons $)$. Monoenergetic, stable, narrow divergence electron beams are produced by LWFAs at the required energies for Thomson gamma sources.

Simulations (VORPAL code [12]) benchmarked to the experiments showed that the production of monoenergetic bunches occurred when the accelerator operated at the threshold of trapping electrons from the plasma. In this case trapping of electrons loaded the wake, preventing further injection [3]. The guiding structure and tuning of the density then allowed the bunch to accelerate until it outran (dephased from) the wake, which condensed the electrons in energy space. The self-trapping, wake generation and guiding were all interdependent in the regime of these recent experiments.

Controlled injection can reduce momentum spread, and increase stability and tunability, by decoupling injection from guiding and wake generation. Recent experiments used plasma density gradients in a gas jet to control the wake phase velocity and trapping 
threshold, producing stable electron bunches with longitudinal and transverse momentum spreads more than 10 times lower than in previous experiments $(0.17$ and $0.02 \mathrm{MeV} / \mathrm{c}$ FWHM, respectively) and with central momenta stable at $0.76 \pm 0.02 \mathrm{MeV} / \mathrm{c}$ over several days [7]. Such a jet has now been embedded in the capillary structure, and the first runs with this setup have already produced increased stability in 0.5 GeV-class bunches [13]. Colliding pulse optical injection can provide additional control, possibly in combination with density gradients [14]. Simulations indicate that control of injection can result in energy spreads and divergences below $1 \%$ and $1 \mathrm{mrad}$ [15].

\section{MONOENERGETIC GAMMA SOURCE}

Thomson scattering has been developed on conventional linacs, including the first demonstration of the generation of sub-picosecond duration X-ray pulses using $90^{\circ} \mathrm{TS}$ at the Beam Test Facility of the Advanced Light Source at LBNL [1]. The gamma energy is given above, and the energy spread and divergence are a convolution of electron beam energy spread and divergence with laser bandwidth and divergence [16]. For narrow bandwidth sources, headon collision with a long pulse laser with $>1 /(\Delta \mathrm{E} / \mathrm{E})$ periods in the pulse is desired. Laser intensity $<10^{18} \mathrm{~W} / \mathrm{cm}^{2}$ is desired to prevent multi-photon scattering and resultant broadening, and these requirements together with the laser and electron beam diffraction ranges set the required laser power. Source parameters have been calculated. As an example, take a $300 \mathrm{MeV}$ electron beam with $10^{9}$ electrons and $2 \%$ energy spread, and with a bunch radius of $10 \mu \mathrm{m}$, similar to recent experiments. A $40 \mathrm{~J}, 13$ ps scattering laser of $1 \mu \mathrm{m}$ wavelength focused to $25 \mu \mathrm{m}$ radius produces $\sim 3 \times 10^{8}$ gamma rays at $1.7 \mathrm{MeV}$, matched to the U235 NRF line (Fig. 3). Gamma bandwidth will be roughly twice the electron bandwidth (Fig. 4). Divergence is $<2 \mathrm{mrad}$. Using electrons of 400-800 $\mathrm{MeV}$ would access photon energies of $5-15 \mathrm{MeV}$ for photofission.

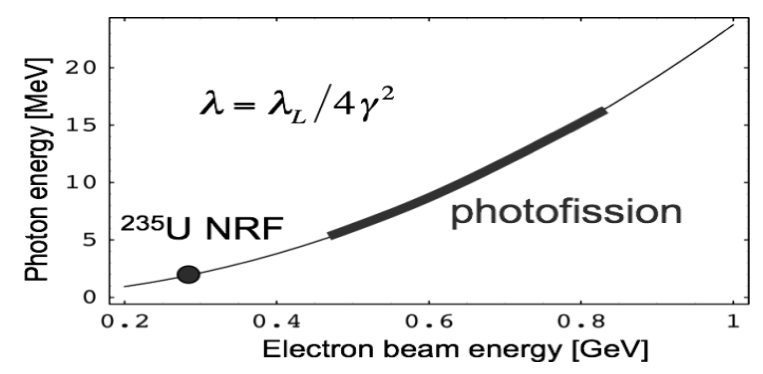

FIGURE 3. Thomson scattering from GeV-class electron bunches, produced by $3-\mathrm{cm}$ LWFAs, produces photon energies in the NRF and photofission windows

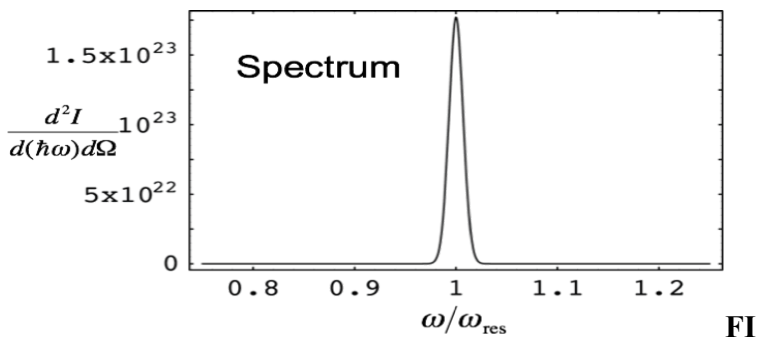

FIGURE 4. Photon spectrum of an LWFA Thomson source in relative frequency (center frequency set by fig. 3). Electron beam used is close to present experiments. $3 \times 10^{8}$ photons can be produced in few \% energy spread from a $40 \mathrm{~J}$ class laser.

\section{CONCLUSION}

Laser wakefield accelerators have demonstrated electron bunches at $0.1-1 \mathrm{GeV}$, and beams with percent stability in energy and mrad stability in pointing. Continued development is using controlled injection to further stabilize and improve performance. It is anticipated that this, together with future laser development, will allow source parameters suitable for SNM applications with $10^{10}$ to more than $10^{12}$ average photons/second at 1.7-15 MeV photon energies, and with the low energy spread desired for NRF applications, in compact sources. The cm-scale accelerator structure could enable steerable sources to allow rastering across targets, which is otherwise difficult.

\section{ACKNOWLEDGMENTS}

This work was supported by the Director, Office of Science, Office of High Energy Physics, of the U.S. Department of Energy under Contracts including No. DE-AC02-05CH11231, DE-FG02-04ER84097, and SciDAC DE-FC02-07ER41499, and by DARPA. The simulations were performed at the National Energy Research Scientific Computing Center (NERSC). We acknowledge the assistance of the VORPAL development team and the contributions of Simon M. Hooker and other colleagues at LOASIS and Oxford. 


\section{REFERENCES}

1. R.W. Schoenlein, W.P. Leemans, et al., Science, 274, (5285), pp. 236 (1996).

2. T. Tajima and J.M. Dawson, Phys. Rev. Lett. 43, pp. 267 (1979).

3. C.G.R. Geddes et al., Nature, 431(7008), pp. 538 (2004).

4. W.P. Leemans, et al., Nature Physics, 2, pp. 696 (2006).

5. C.B. Schroeder, et al., Proc. FEL (2006).

6. J. van Tilborg et al., Opt. Lett. 32 (3), pp. 313 (2007).

7. C.G.R. Geddes et al., PRL V 100, 215004 (2008).

8. LOASIS facility: http://loasis.lbl.gov/.

9. S. P. D. Mangles et al., Nature 431, pp. 535 (2004); also J. Faure et al., Nature 431, pp. 541 (2004).

10. D.J. Spence, et al., J. Phys. B 34 (21), 4103 (2001).

11. K. Nakamura, et al., Proc. Adv. Accel. Concepts Wkshp. (2008, in press).

12. C. Nieter and J. Cary, J. Comp. Phys. 196, 448 (2004).

13. A.J. Gonsalves, et al., (in preparation).

14. E. Esarey et al., Phys. Rev. Lett. 79, 2682 (1997).

15. C.G.R. Geddes, et al., J. Phys. Conf. Series 125, pp. 12002 (2008).

16. W.P. Leemans, et al., IEEE Trans. Plasma Sci. 33(1), pp. 8 (2005). 